\title{
Clustering and phase resetting in a chain of bistable nonisochronous oscillators
}

\author{
V. I. Nekorkin* and V. A. Makarov ${ }^{\dagger}$ \\ Radiophysical Department, Nizhny Novgorod State University, 23 Gagarin Avenue, 603600 Nizhny Novgorod, Russia \\ M. G. Velarde \\ Instituto Pluridisciplinar, Universidad Complutense, Paseo Juan XXIII, No. 1, Madrid 28 040, Spain
}

(Received 10 March 1998; revised manuscript received 16 June 1998)

\begin{abstract}
We consider a chain with many locally coupled bistable nonisochronous oscillators. We show that on the "background" of a disordered amplitude distribution either phase or frequency clusters form in the chain. Cluster location varies according to the amplitude distribution. In the case of frequency clusters their interaction leads to phase resetting in an isolated oscillator located between two neighboring frequency clusters.
\end{abstract} [S1063-651X(98)15611-8]

PACS number(s): $05.45 .+\mathrm{b}$

\section{INTRODUCTION}

Clustering, i.e., formation of groups of elements with identical (or almost identical) properties (amplitudes, phases, or frequencies) is an important phenomenon in nature. In particular, in neurophysiology, recent experimental data on the activity of the visual cortex of cats and monkeys [1] show that stimulation of the retina by a pattern leads to coherent, synchronous firing of spatially separated neurons. These neurons form coherent zones (clusters). Different visual objects excite different zones. Inside each zone oscillations of neurons are synchronous, while oscillations from different clusters are not synchronized among themselves. Coherence in a cluster and time-binding of different albeit related clusters seems to underlie perception and processing of the information by the brain $[2,3]$.

Neural networks in nature are extremely complex for the mathematical description, hence, there is the need for considering rather simple models to understand or explain the various features of brain activity [4]. For example, it is known that formation of clusters occurs in network systems with global coupling between elements [5-9], in systems with pulse-coupled oscillators having time delays [10], in time-delayed nearest-neighbor coupled phase oscillators [11], in stochastically coupled networks [12], in systems of locally interacting phase oscillators $[13,14]$, etc. Here we study cluster formation in a chain of locally coupled (nearest neighbors) bistable oscillators. Such a model may be useful to describe the phenomena of time binding of several spatially divided visual images. Each image will fire its own group of oscillators. In our model an individual neuron may be in two modes: at rest or periodically oscillating (limit cycle) [15]. We prove that stable phase and frequency clusters can exist in a system with diffusive coupling provided two basic conditions are fulfilled. These conditions are:

(i) The amplitude distribution is chaotic along the chain.

(ii) Each unit of the chain is a nonisochronous oscillator,

\footnotetext{
*Electronic address: nekorkin@rf.unn.runnet.ru
${ }^{\dagger}$ Electronic address: makarov@ @ale.appl.sci-nnov.ru

*Electronic address: nekorkin@rf.unn.runnet.ru
†Electronic address: makarov@hale.appl.sci-nnov.ru

"Electronic address: mvelarde@eucmax.sim.ucm.es
}

.

i.e., the oscillation frequency of each unit depends on its amplitude [16].

Note that for cluster formation these conditions must be simultaneously satisfied. For example, if a chain consists of isochronous self-excited units, then formation of (phase) clusters is a transient process only $[17,18]$. After clusters form, their interaction is self-destructive, leading to in-phase oscillations along the chain.

To illustrate the phenomena just described, let us consider an oscillator obeying the equations in polar coordinates,

$$
\begin{aligned}
& \dot{r}=r F(r), \\
& \dot{\varphi}=\omega(r),
\end{aligned}
$$

where $r, \varphi$ are the oscillation amplitude and phase, respectively, $\omega(r)=\alpha r^{2}$ is the angular frequency of oscillations, which depends on its amplitude, and $r F(r)$ is a nonlinear function having three zeros (Fig. 1). Equations (1) describe a bistable, nonisochronous oscillator that can be either at rest (stable steady state) or in the oscillatory state (stable limit cycle). Then for a chain consisting of $N$ oscillators with diffusive nearest-neighbor coupling the equations are

$$
\begin{gathered}
\dot{z}_{j}=z_{j}\left[F\left(\left|z_{j}\right|\right)+i \omega\left(\left|z_{j}\right|\right)\right]+d\left(z_{j-1}-2 z_{j}+z_{j+1}\right), \\
j=1,2, \ldots, N, \quad z_{0}=z_{1}, \quad z_{N+1}=z_{N},
\end{gathered}
$$

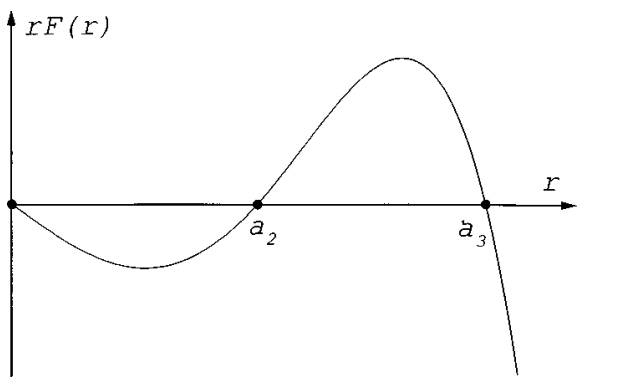

FIG. 1. Function characterizing the dynamics of a bistable unit or element in the chain. Units are arbitrary. 
where $z_{j}=r_{j} e^{i \varphi_{j}}$ and $d$ accounts for the local diffusive coupling between oscillators ( $d$ is real and positive). The index $j$ stands for the variables of the $j$ th unit of the chain.

The model (2) belongs to a class of weakly coupled spatially distributed oscillatory systems. Spatiotemporal dynamics of such chains and lattices have been discussed by several authors. The dynamics of networks where each site has a limit cycle oscillator (in our case it depends on the nonlinear function $F$ ) can be reduced to the investigation of networks of phase oscillators [19]. In Ref. [20] the investigation of phase waves has been carried out in a chain of oscillators when the attractor is in a limit cycle close to a Hopf bifurcation. In Ref. [13] it was shown that a model of interacting phase oscillators with random intrinsic frequencies shows a spongelike cluster structure. The estimation of the critical dimension of a $d$-dimensional lattice necessary for macroscopic mutual entrainment was obtained in [21]. The influence of coupling schemes in two-dimensional (2D) lattices of phase oscillators with random intrinsic frequencies on robust phase locking oscillations was investigated in [22]. It was found that sparse coupling leads to a more rapid and robust phase locking than nearest-neighbor coupling. In Ref. [14] mutual entrainment in lattices with nonvariational interaction was discussed. Many other publications exist that are devoted to the study of the evolution of phase oscillators with global coupling (see, for example, [5-9,23], and references therein).

The behavior of systems of multistable oscillators [in our case it corresponds to a nonlinear function $r F(r)$ having more than two zeros] or with spatially nonuniform units is not as frequently investigated. For instance, in [24] the propagation of fronts in a chain of bistable oscillators was considered. The criterion for the existence of localized oscillations, so-called discrete breathers in a network where each site has an equilibrium or steady state and some sites have a limit cycle, was formulated in [25]. Domains of existence of spatial chaos for 1D and 2D networks of bistable oscillators were found in [17] and [18], respectively.

In Sec. II we deal with the existence of spatial chaos in the distribution of amplitudes along the chain. In Sec. III we consider the chain (2) with units weakly nonisochronous. We show how stable phase clusters form. Then in Sec. IV we show how frequency clusters are produced when the units are drastically nonisochronous. We also study the phenomenon of phase resetting. In Sec. V we summarize our results.

\section{AMPLITUDE DISTRIBUTION ALONG THE CHAIN}

It is shown in the Appendix that for parameter values belonging to a certain region $D_{\mathrm{ch}}$ [see inequality (A5)] in the phase space of the system (2) there are $2^{N}$ invariant domains. Figure 2 illustrates the region, $D_{\mathrm{ch}}$, corresponding to the choice $F(r)=-2 a r^{4}+a r^{2}-1$. These invariant domains are intersections of the following regions:

$$
\begin{aligned}
& V_{j}^{0}=\left\{0 \leqslant r_{j} \leqslant b_{0}, \quad 0 \leqslant r_{k} \leqslant B, \quad \forall k, \quad k \neq j\right\}, \\
& V_{j}^{1}=\left\{b_{1} \leqslant r_{j} \leqslant B, \quad 0 \leqslant r_{k} \leqslant B, \quad \forall k, \quad k \neq j\right\},
\end{aligned}
$$

where $b_{0}, b_{1}$, and $B$ are certain constants that depend on $d$ and the specific nonlinear function $F(r)$. The vector field of

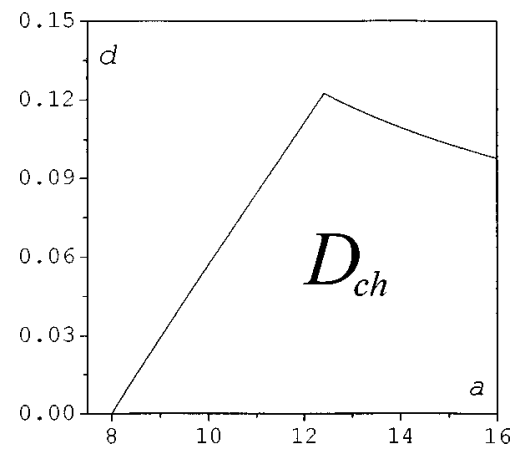

FIG. 2. Domain, $D_{\text {ch }}$, of the amplitude disorder along the chain for $r F(r)=-2 a r^{5}+a r^{3}-r$. Quantities are dimensionless.

the system (2) at the boundary of the regions $V_{j}^{0}$ and $V_{j}^{1}$ is oriented toward these regions. Consequently, if the initial amplitudes, $\left\{r_{j}(0)\right\}$, belong to the regions $V_{j}^{0}$ or $V_{j}^{1}$, then the $\left\{r_{j}(t)\right\}$ are located, remain forever inside these regions, and remain small $\left(V_{j}^{0}\right)$ or large $\left(V_{j}^{1}\right)$. Thus, for points of the parameter region $D_{\text {ch }}$ there exist at least $2^{N}$ different distributions of the amplitudes of oscillations along the chain (2). Since each distribution can be "coded" by an arbitrary sequence of two symbols [17] the distribution of the amplitudes can be very diverse, including regular and disordered spatially chaotic distributions.

\section{PHASE CLUSTERS}

Let us first start with $\alpha$ small enough. To get a disordered amplitude distribution along the chain we choose the initial amplitudes, $\left\{r_{j}(0)\right\}$, from either $V_{j}^{0}$ or $V_{j}^{1}$. The initial phases are randomly distributed in the range $[-\pi, \pi]$. The numerical integration of (2) shows that the process of formation of stationary amplitude and phase distributions occurs in two stages with different time scales. First, there is a short stage at which the equilibrium amplitude distribution $\left(r_{j}=r_{j}^{0}\right.$, where $r_{j}^{0}$ are constants) is formed [Fig. 3(a)]. Then, slowly, phase clusters form along the chain [Figs. 3(b) and 3(c)]. The number and spatial location of clusters are determined by the former amplitude distribution. The chain splits into phase clusters at the sites of gaps in the amplitude distribution (i.e., at the sites where oscillation amplitudes, $r_{j}$, are small). In Fig. 3(b) three phase clusters are shown: PC1 (oscillators at sites 1-10), PC2 (oscillators at sites 12-27), PC3 (oscillators at sites 28-36), and one oscillator (at site 11) that does not belong to these clusters. Inside each cluster, the phase differences, $\psi_{j}=\varphi_{j+1}-\varphi_{j} \simeq 0$, are very small but between clusters there are sharp jumps in phase, i.e., $\psi_{10}, \psi_{11}$, and $\psi_{27}$ are nonzero constants.

As in the chain a phase cluster mode corresponds to the quantities $r_{j}, \psi_{j}$ being constant, then it corresponds to a steady state in the phase space of the system (A1). From the equations for the phase differences, $\sin \psi_{j}$, we have a system of $(N-1)$ linear equations. Its solution is

$$
\sin \psi_{j}=\frac{\alpha\left(\sum_{i=1}^{j} r_{i}^{2} \times \sum_{i=j+1}^{N} r_{i}^{4}-\sum_{i=j+1}^{N} r_{i}^{2} \times \sum_{i=1}^{j} r_{i}^{4}\right)}{d r_{j+1} r_{j} \sum_{i=1}^{N} r_{i}^{2}} .
$$




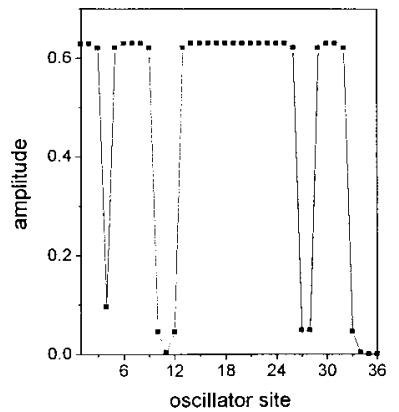

(a)

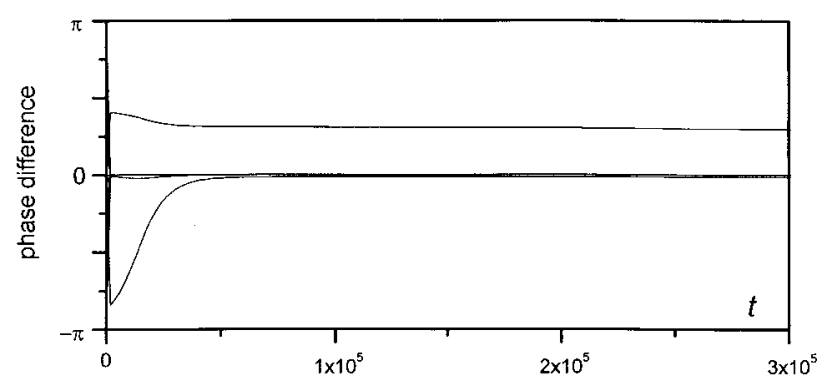

(c)

FIG. 3. Formation of phase clusters $(\alpha=0.0018, d=0.08, a$ =12). (a) Final amplitude distribution, (b) snapshot of the distribution of phases along the chain, (c) phase differences $\left(\psi_{j}=\varphi_{j+1}\right.$ $\left.-\varphi_{j}\right)$ vs time. Quantities are dimensionless.

From (4) it follows that the differences in phase between neighboring oscillators are proportional to $\alpha / d$ and depend on the chosen distribution of amplitudes. As $\sin \psi_{j}$ $\sim \alpha\left(d r_{j} r_{j+1}\right)^{-1}$ the sharp phase jump between neighboring oscillators can be observed in positions where gaps in amplitude distribution appear, i.e., when $r_{j}, r_{j+1}$ have low values, hence the splitting of the chain into phase clusters. Figure 3(a) shows three such gaps where the amplitudes of neighboring oscillators, $r_{j}, r_{j+1}$, are small. They appear at sites 10, 11, 12, at sites 27, 28, and at sites 33-36. In Fig. 3(b) we see that there is a small phase shift between clusters in oscillators at sites 27,28 . The largest phase shifts are observed between oscillators at sites 10,11 and 11, 12 [Fig. 3(b)]. This comes from the fact that the values $\left(r_{10} r_{11}\right)$ and $\left(r_{11} r_{12}\right)$ are much smaller than $\left(r_{27} r_{28}\right)$. The lack of splitting into clusters in the gap at sites $33-36$ is due to the compensation of the sums contained in Eq. (4). Thus, although the distribution of amplitudes and phases along the chain is rather complex and the frequency of each unit, taken separately, depends on its amplitude, phase clustering appears.

\section{FREQUENCY CLUSTERS AND PHASE RESETTING}

\section{A. Clustering and phase resetting}

Let us now consider $\alpha$ large enough, e.g., so large that phase clusters cannot be maintained in the chain. As in the preceding section, we choose initial conditions for amplitudes $\left\{r_{j}\right\}$ belonging to the regions $V_{j}^{0}$ or $V_{j}^{1}$. Numerical simulations show that after a transient stage a certain distribution of amplitudes along the chain is established. In this case the amplitudes are not constants, but they are bounded and remain inside the regions $V_{j}^{0}, V_{j}^{1}$. Figure 4(a) is a snap-

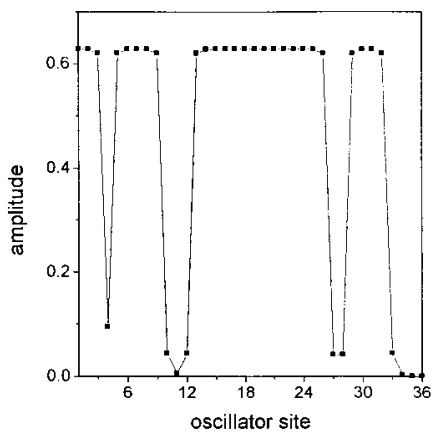

(a)

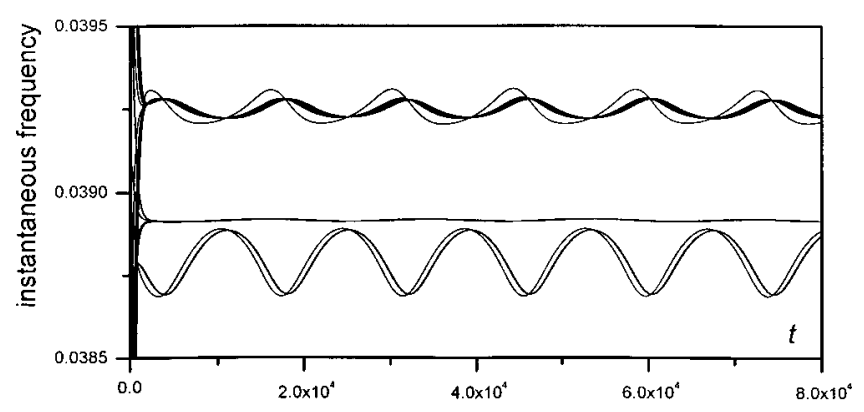

(b)

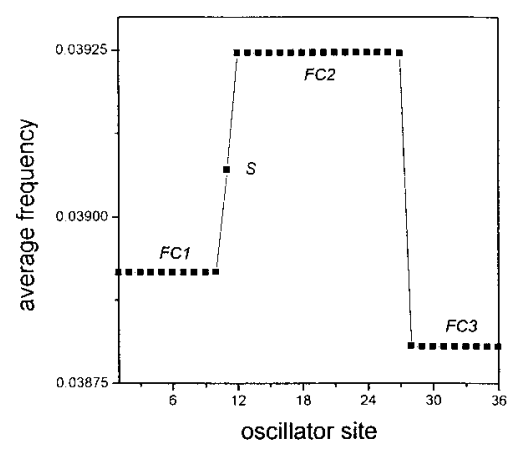

(c)

FIG. 4. Formation of frequency clusters $(\alpha=0.1, d=0.08, a$ $=12$ ). (a) Snapshot of the amplitude distribution, (b) instantaneous frequencies $\left[\omega_{j}(t)=\dot{\varphi}_{j}\right]$ vs time, (c) average frequency along the chain. Quantities are dimensionless.

shot of the amplitude distribution along the chain. It is very similar to the stationary amplitude distribution shown in Fig. 3(a).

Let us now consider the behavior of phases of the oscillators and their frequencies $\omega_{j}=\dot{\varphi}_{j}$. Figure 4(b) shows the behavior of the instantaneous frequencies of oscillations. The formation of three groups of such instantaneous frequencies is a characteristic property of the chain in this case [Fig. 4(b)]. Each frequency group has a different number of oscillators. For each of these groups an average frequency can be defined. Figure 4(c) shows the distribution of average frequencies along the chain. We see that the chain splits into three frequency clusters: FC1 (oscillators at sites 1-10), FC2 (oscillators at sites 12-27), FC3 (oscillators at sites 28-36), and one isolated oscillator at site 11 (further, we call it $S$ ). Let us denote the average frequencies of FC1, FC2, and FC3 with $\Omega_{1}, \Omega_{2}$, and $\Omega_{3}$, respectively. Note that in each frequency cluster there are oscillators with low and high ampli- 


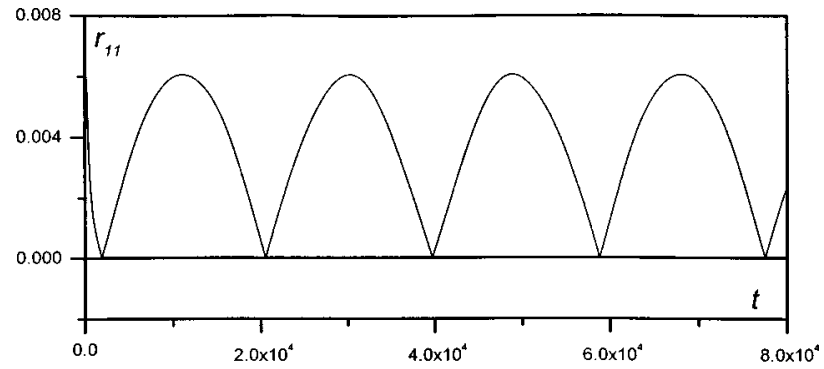

(a)

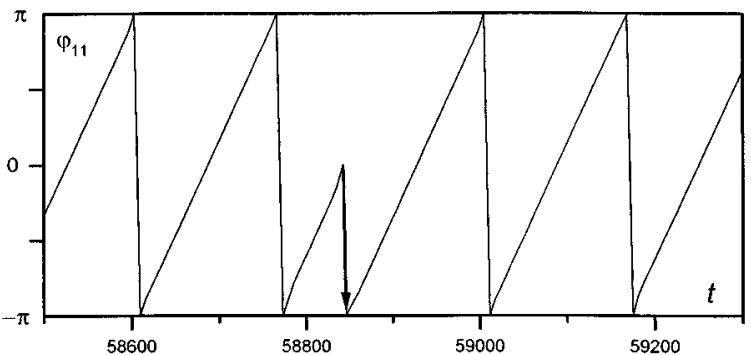

(b)

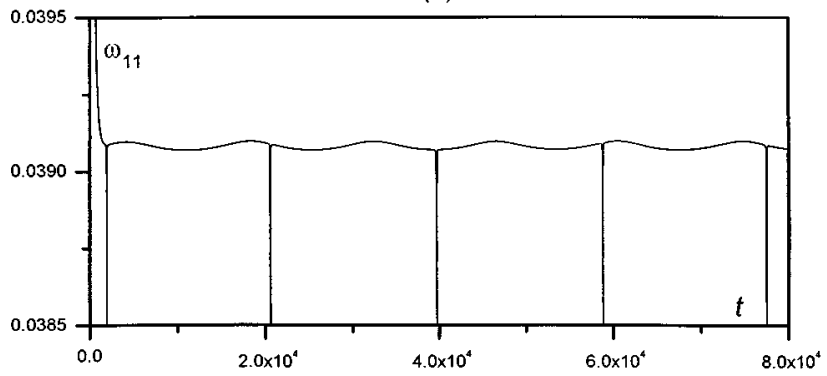

(c)

FIG. 5. Phase resetting. All conditions correspond to the formation of frequency clusters (Fig. 4). (a) Amplitude of oscillation at site (11) vs time, (b) phase resetting process for oscillator at site (11) (a different time scale was used), (c) instantaneous frequency of oscillations at site (11) vs time, singularities of frequency. Quantities are dimensionless.

tudes of oscillations. Comparing Figs. 3(b) and 4(c) we see that splitting into clusters takes place in the same spots both in phase and in frequency cases, namely, where amplitude gaps exist (at sites 10-12 and 27, 28). Thus, basically, cluster formation in the chain is a similar process for phase clusters and frequency clusters.

Let us now investigate the behavior of the oscillator $S$ in further detail. The time evolution of amplitude $r_{11}(t)$, of phase $\varphi_{11}(t)$, and of frequency $\omega_{11}(t)$ are shown in Figs. 5(a), 5(b), and 5(c), respectively. From time to time the function $r_{11}(t)$ vanishes [Fig. 5(a)]. At these instants of time we see very short peak singularities in the time dynamics of the instantaneous frequency $\omega_{11}$ [see Fig. 5(c)]. Simultaneously, phase $\varphi_{11}(t)$ jumps of $\pi$ occur [arrow on Fig. 5(b)]. Then there is phase resetting in the chain [26]. Phase resetting is the result of the complex collective dynamics of the chain. During the time evolution three different populations of oscillators (frequency clusters) are formed in the chain. The oscillator $S$ is affected by these clusters. FC1 and FC2 try to "enslave" this isolated element. In such a competition process none of the clusters is a winner. The oscillator $S$ keeps its average frequency. However, from time to time it is forced to change its phase by a value $\pi$, so that the parity between clusters FC1 and FC2 is maintained.

\section{B. Phenomenological model of phase resetting}

Let us consider oscillators belonging to the frequency cluster $\mathrm{FC} 1$ as a single element that rotates with angular frequency $\Omega_{1}$, and the oscillators belonging to FC2 as another element with frequency $\Omega_{2}$. They are independent and act on oscillator $S$. Comparing Figs. 4(b) and 5(c) we see that the instantaneous frequency of the oscillator $S$ is between the frequencies of FC1 and FC2. Let the frequency of the oscillator $S$ be $\Omega_{s}=\left(\Omega_{2}+\Omega_{1}\right) / 2$. Let us further consider the case of equality of initial phases of clusters and of the oscillator $S$. Moreover, since the amplitude of oscillations of $S$ is small we expand the nonlinear function $r F(r)$ and omit all terms beyond first order, hence $r F(r) \approx-r$. Then, from Eqs. (A1) we get a single equation describing the time evolution of the amplitude of $S$,

$$
\dot{r}=-(1+2 d) r+d\left(R_{1}+R_{2}\right) \cos \frac{\Omega_{2}-\Omega_{1}}{2} t,
$$

where $R_{1}$ and $R_{2}$ are constants characterizing the interaction between the clusters and the oscillator $S$. Equation (5) is a linear ordinary differential equation whose solution is

$$
r(t)=C e^{-\gamma t}+\frac{d\left(R_{1}+R_{2}\right)}{(\Delta \Omega)^{2}+\gamma^{2}}(\gamma \cos \Delta \Omega t+\Delta \Omega \sin \Delta \Omega t),
$$

where $C$ is an arbitrary constant, $\Delta \Omega=\left(\Omega_{2}-\Omega_{1}\right) / 2$, and $\gamma$ $=1+2 d$. From (6) it follows that $r(t)$ is a function that almost periodically reaches zero and, eventually, reverses its sign. As $r(t)$ corresponds to the amplitude of oscillation of the oscillator $S$, inversion of the sign corresponds to a $\pi$ change in the phase of oscillations in the oscillator $S$, hence, phase resetting. The period, $T$, between successive zeros of the function $r(t)$ can be estimated from (6),

$$
T=\frac{2 \pi}{\Omega_{2}-\Omega_{1}} .
$$

Taking the values of $\Omega_{1}$ and $\Omega_{2}$ from Figs. 4(c) to (7) we get $T \cong 1.9 \times 10^{4}$. This value agrees satisfactorily with the period of frequency peaks observed in Fig. 5(c).

\section{CONCLUSION}

We have investigated processes of formation of phase and frequency clusters that may be relevant to understanding basic features of synchronization of neuron oscillations in brain activity. Coexistence of chaos and order is fundamental in the dynamics of a chain of many locally, diffusively coupled bistable nonisochronous oscillators. Indeed, if the amplitude distribution of oscillations along the spatial coordinate $j$ is chaotic, then such a distribution can be the background for clustering. In spite of spatially chaotic amplitudes, the oscillators take on regular temporal patterns: phase and frequency clusters. Choosing definite initial conditions for amplitudes of oscillations from domains $V_{j}^{0}$ or $V_{j}^{1}$ we can prescribe a given final distribution of $r_{j}$, which in turn determines spa- 
tial areas of synchronous oscillations. In other words, the stimulation of various oscillating neurons results in the formation of synchronous zones appropriate for an initial patterned stimulus. We note that as the zone sites are defined by amplitude distribution, it is stable to rather large perturbations, and can be changed by the action of a new stimulus which will transfer the system to another invariant domain.

In addition, we have found that the competition between frequency clusters is the basis for the phenomenon of phase resetting. It appears when two conditions are fulfilled: (i) There are two (or more) frequency clusters in the chain, and (ii) a single oscillator appears isolated between them. Cluster competition yields jumps of $\pi$ in the phase of the isolated oscillator.

Finally, we have recently learned about the experimental work by Llinás and Yarom [27] on the oscillatory properties of guinea-pig inferior olivary (i.o.) neurons. They showed that in vitro and in vivo i.o. neurons possess intrinsic mechanisms which allow them to function on the one hand as autonomous oscillators and on the other hand as a synchronized neuronal ensemble. They also showed that i.o. neurons change their oscillatory behavior in the presence of certain pharmacological substances. Their data reflect the properties of a large number of coupled units. In particular, their Fig. 5(D) [27] shows a case of phase resetting in perfect agreement with one of our findings, Fig. 5(b).

\section{ACKNOWLEDGMENTS}

The authors acknowledge fruitful discussions with R. Llinas, R. MacKay, P. Coullet, A. Fdez. de Molina, and L. O. Chua. This research has been supported by the BCH Foundation, by the Russian Foundation for Basic Research under Grant No. 97-02-16550, by DGICYT under Grant No. PB 96-599, and by NATO under Grant No. OUTR LG 96-578. V.I.N. would like to acknowledge the Ministry of Education (Spain) for economic support.

\section{APPENDIX: CONSTRUCTION OF INVARIANT DOMAINS}

Let us take Eqs. (2) using amplitudes and phase shifts $\left(\psi_{j}=\varphi_{j+1}-\varphi_{j}\right)$ :

$$
\begin{gathered}
\dot{r}_{j}=r_{j} F\left(r_{j}\right)+d\left(r_{j-1} \cos \psi_{j-1}-2 r_{j}+r_{j+1} \cos \psi_{j}\right), \\
r_{k} r_{k+1} \dot{\psi}_{k}=r_{k} r_{k+1}\left[\omega\left(r_{k+1}\right)-\omega\left(r_{k}\right)\right]+d\left(r_{k} r_{k+2} \sin \psi_{k+1}\right. \\
\left.-\left(r_{k}^{2}+r_{k+1}^{2}\right) \sin \psi_{k}+r_{k+1} r_{k-1} \sin \psi_{k-1}\right), \\
j=1,2, \ldots, N, \quad k=1,2, \ldots, N-1, \\
r_{0}=r_{1}, \quad r_{N+1}=r_{N}, \quad \psi_{0}=0, \quad \psi_{N}=0 .
\end{gathered}
$$

\section{Location of the bounded solutions $\left\{r_{j}(t)\right\}$ in the phase space}

Let us consider in the phase space of the system (A1) the following regions:

$$
V_{B}=\left\{\mathbf{r}: 0 \leqslant r_{j} \leqslant B, \quad j=1,2, \ldots, N\right\},
$$

where $B$ is a parameter. From (A1) and the form of $r F(r)$ (Fig. 1) it follows that at $B>a_{3}$ on the boundary of each region $V_{B}$ the vector field of (A1) points into $V_{B}$. Now we consider the orientation of the vector field on the surfaces $\left\{r_{j}=0\right\}$. As for $r_{j}=0$ the value of the phase $\varphi_{j}$ is left undetermined; we take $\varphi_{j}$ such that

$$
\dot{r}_{j \mid r_{j}=0}=d\left(r_{j-1} \cos \psi_{j-1}+r_{j+1} \cos \psi_{j}\right) \geqslant 0 .
$$

Hence, the vector field of the system (A1) is brought inside $V_{B}$ and all bounded solutions $\left\{r_{j}(t)\right\}$ are located in the region $V_{B}$, where $B=a_{3}$.

\section{Invariant domains}

Following the method discussed in [18] we construct in the phase space of the system (A1) a family of narrower invariant domains. For this purpose we consider the orientation of the vector field of (A1) on surfaces $\left\{r_{k}=c\right\}$, where $c=$ const $<a_{3}$. From (A1) we obtain

$$
\dot{r}_{k \mid r_{k}=c}=c F(c)+d\left(r_{k-1} \cos \psi_{k-1}-2 c+r_{k+1} \cos \psi_{k}\right) \text {. }
$$

Let us demand that

$$
\dot{r}_{\left.k\right|_{r_{k}=c}}<0 \quad \text { for } \quad r_{j} \in V_{B}, \quad \forall{ }_{j} \neq k
$$

This condition is satisfied for the parameter region, $D_{\mathrm{ch}}$, defined by the inequality

$$
D_{\mathrm{ch}}=\left\{d<\min \left(\frac{r_{\max } F\left(r_{\max }\right)}{2\left(a_{3}+r_{\max }\right)}, \frac{\left|r_{\min } F\left(r_{\min }\right)\right|}{2\left(a_{3}-r_{\min }\right)}\right)\right\},
$$

where $r_{\text {min,max }}$ are coordinates of the minimum and of the maximum of $r F(r)$. In this case $c=b_{0}$, where $b_{0}$ is the smallest root of the equation

$$
c F(c)-2 d\left(c-a_{3}\right)=0 .
$$

Similarly, for points of $D_{\mathrm{ch}}$ the inequality

$$
\dot{r}_{\left.k\right|_{r_{k}=c}}>0 \quad \text { for } \quad r_{j} \in V_{B}, \quad \forall j \neq k
$$

is satisfied when $c=b_{1}$, where $b_{1}$ is the largest root of the equation

$$
c F(c)-2 d\left(c+a_{3}\right)=0 .
$$

Using the surfaces $\left\{r_{k}=b_{0}\right\},\left\{r_{k}=b_{1}\right\}$, and the boundary of the domain $V_{B}$, we can introduce domains $V_{j}^{0}$ and $V_{j}^{1}$ [see Eq. (3)]. Then the vector field of the system (A1) at the border of the regions $V_{j}^{0}, V_{j}^{1}$ is oriented toward these regions. Thus, for the points of the parameter region $D_{\mathrm{ch}}$ there exist $2^{N}$ invariant domains that are intersections of the domains $V_{j}^{0}, V_{j}^{1}$. 
[1] P. Koening et al., Eur. J. Neurosci. 5, 501 (1993); see also J. Krueger and F. Aiple, J. Neurophysiol. 60, 798 (1988).

[2] R. Llinas and U. Ribary, Proc. Natl. Acad. Sci. USA 90, 2078 (1993)

[3] H. Haken, Principles of Brain Functioning (Springer-Verlag, Berlin, 1996).

[4] H. D. I. Abarbanel, M. I. Rabinovich, A. Selverston, M. V. Bazhenov, R. Huerta, M. M. Sushchik, and L. L. Rubchinskii, Usp. Fiz. Nauk 166, 1 (1996) [Phys. Usp. 39, 337 (1996)].

[5] K. Kaneko, Physica D 75, 74 (1994).

[6] V. Hakim and W.-J. Rappel, Phys. Rev. A 46, R7347 (1992).

[7] N. Nakagawa and Y. Kuramoto, Physica D 75, 74 (1994); see also 80, 307 (1995).

[8] P. Tass and H. Haken, Z. Phys. B 100, 303 (1996); see also P. Tass, Phys. Rev. E 56, 2043 (1997).

[9] K. Okuda, Physica D 63, 424 (1993).

[10] U. Ernst, K. Pawelzik, and T. Geisel, Phys. Rev. Lett. 74, 1570 (1995).

[11] Y. Nakamura, F. Tominaga, and T. Munakata, Phys. Rev. E 49, 4849 (1994).

[12] J. Lin, K. Pawelzik, U. Ernst, and T. Sejnowski (unpublished).

[13] S. H. Strogatz and R. E. Mirollo, J. Phys. A 21, L699 (1988).

[14] H. Sakaguchi, S. Shinomoto, and Y. Kuramoto, Prog. Theor. Phys. 79, 1069 (1988).

[15] Coupled Oscillating Neurons, edited by J. G. Taylor and C. L.
T. Mannion (Springer-Verlag, Berlin, 1992).

[16] A. A. Andronov, A. A. Vitt, and S. E. Khaikin, Theory of Oscillations (Pergamon, New York, 1966).

[17] V. I. Nekorkin and V. A. Makarov, Phys. Rev. Lett. 74, 4819 (1995).

[18] V. I. Nekorkin, V. A. Makarov, V. B. Kazantsev, and M. G. Velarde, Physica D 100, 330 (1997).

[19] A. T. Winfree, J. Theor. Biol. 16, 15 (1967); see also G. B. Ermentrout and J. Rinzel, J. Math. Biol. 11, 269 (1981); see also Y. Kuramoto, Chemical Oscillations, Waves, and Turbulence (Springer-Verlag, Berlin, 1984).

[20] G. B. Ermentrout, J. Math. Biol. 23, 55 (1985).

[21] H. Daido, Phys. Rev. Lett. 61, 231 (1988).

[22] E. Niebur, H. G. Schuster, D. M. Kammen, and C. Koch, Phys. Rev. A 44, 6895 (1991).

[23] G. B. Ermentrout, Physica D 41, 219 (1990); see also H. Daido, Phys. Rev. Lett. 73, 760 (1994).

[24] A.-D. Defontaines, Y. Pomeau, and B. Rostand, Physica D 46, 201 (1990).

[25] J.-A. Sepulchre and R. S. MacKay, Nonlinearity 10, 679 (1997).

[26] A. T. Winfree, The Geometry of Biological Time (SpringerVerlag, New York, 1980).

[27] R. Llinas and Y. Yarom, J. Physiol. (London) 376, 163 (1986). 